Zitate von VC III 13f. und 21f. sind aus der Hist. Eccl. Theodorets abgeschrieben. 1

Auch die nur in einer Handschrift, dem Athener Cod. suppl. gr. $2560 \quad 369 \mathrm{c}$ (olim Serres membr. A 40) ${ }^{2}$, überlieferte Vita BHG Nr. 369c hat nur geringe Bedeutung für unsere Zwecke. Es sind zwar einige kurze, inhaltliche Parallelen zur VC zu finden, doch ist die VC selbst nicht benutzt. Nur an wenigen Stellen des Sachapparates ist die Vita deshalb erwähnt, zitiert nach den Folien der Handschrift.

Nur zu einer Stelle der VC (S. 55, 16-18) fand ich in dem Logos zur 410 Kreuzauffindung des Alexander Monachos (BHG Nr. 410) eine sehr entfernte Parallele ${ }^{3}$.

Natürlich habe ich nur die für die Textkonstituierung relevanten $\mathrm{Ab}$ weichungen dieser Viten von dem VC-Text in den textkritischen Apparat dieser Edition aufgenommen. Zum großen Teil läßt sich nämlich erkennen, wo ein Eingriff der Hagiographen vorliegt. Entweder machen sie erläuternde oder panegyrische Zusätze oder erleichtern schwierige Perioden der VC oder ändern in späterer Zeit ungebräuchliche Worte und Begriffe.

\title{
Der Wert der Überlieferung ${ }^{4}$
}

Die Überlieferung läßt sich durch das folgende Stemma anschaulich machen:

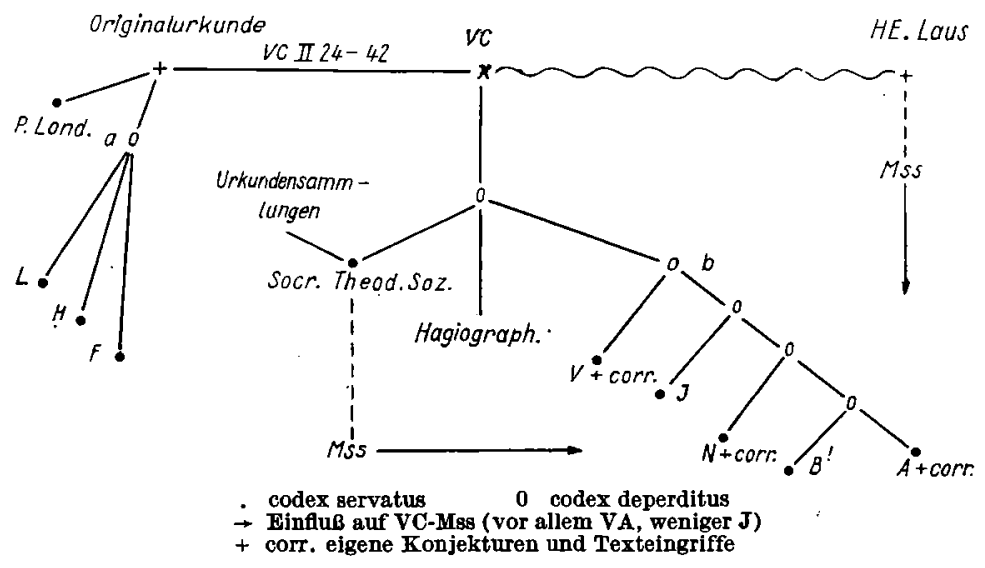

1 Vgl. F. Winkelmann, Das Enkomium auf die 318 nicänischen Väter, Eirene 4, 1965, 39-41.

2 Vgl. Ehrhard I 3, 798.

3 Die Edition dieses hagiographischen Textes von Pio Carmelo Pennacchini (Discorso storico dell'invenzione della Croce del Monaco Alessandro, Grottaferrata 1913, 7-75) führt über die alte von Jacob Gretser (Abdruck Migne PG 87, 3) nicht hinaus.

4 Vl. zum Stemma TU 84, S. 164. S. 117-163 sind Wert und Charakter der wichtigen Textzeugen detailliert untersucht. 
Mit unseren Textzeugen ist es nicht möglich, einige Lücken im Text, teilweise beträchtlichen Umfanges, zu schließen: S. 65, 4 (von $a$ als Lücke gekennzeichnet); 86,$15 ; 134,24 ; 135,1$ (vermutete Lücken); 144, 1 (von $b$ als Lücke gekennzeichnet) 1 .

Die Urkunde VC II 24-42 ruht auf einer besseren Überlieferung als der übrige Text. An 114 Stellen wurde die Gruppe $a$ der geschlossenen Gruppe $b$ vorgezogen: S. 58, 15. 20 (bis). 22. 23 (bis). 29; 59, 3. 12 (bis). 17. 18. 19. 25; 60, 3. 5 (bis). 6 (bis). 7. 10. 12 (bis). 14. 15. 16. 17; 61, 2. 6 (bis). 9. 11 (bis). 12.13. 14. 15. 19. 20. 21. 22. 23; 62,1. 3 (ter). 8. 15. 16 (bis). 18 (bis). 23.24 (bis); 63,1. 4. 5. 8. 10 (bis). 13.14 (ter). 15. 17. 23 (bis). 24. 25; 64,1.4 (bis). 5.7 (bis). 8. 12. 15 (bis). 16 (bis). 17. 18 (bis). 19 (bis). 21. 22. 23. 24 ; 65, 1.13. 15 (bis). 18 (bis). 20. 24. 25. 26. 27 (bis); 66, 1 (bis). 2 (ter). 5. 7. 9 (bis). 11. Doch auch die Gruppe $a$ ist von Fehlern nicht frei. In zwei Fällen sind $a$ und $b$ gemeinsam falsch: S. 62,24 und 64,6. An mehreren Stellen wurden Lesarten von $a$ verworfen: S. 58, 8; 59,10. $15(a \mathrm{~J}) .25$; 60,1 . 9. 23. $24 ; 61,4 ; 63,16(a \mathrm{~J}) ; 64,9.10 .15$ (bis). $16(a \mathrm{~J})$ ) 17. 22 $\left(a \mathrm{~B}^{\mathrm{c}}\right) .23$ (bis); 65, 3. $7(a \mathrm{~J}) .19 ; 66,1(a \mathrm{~J}) .4$. Einige Male wird $a$ durch $J$ gestützt. Die Güte der Überlieferung durch die Gruppe $a$ wird auch durch den Pap. Lond. 878 bestätigt.

Die Parallelurkunden in den Werken der Kirchenhistoriker sind im Vergleich damit nicht so gut zu beurteilen. Einmal sind sie anderen Urkundensammlungen entnommen, als sie Euseb vorlagen, zum anderen ist ihre Überlieferung nicht so einwandfrei, daß sie den VCHandschriften unbedingt überlegen wäre. Nur bei Differenzen innerhalb der Gruppe $b$ und besonders bei Fehlern der ganzen Gruppe haben sie Bedeutung. Der letzte Fall tritt an den folgenden Stellen auf: S. $78,11.22 ; 91,10.15 ; 92,19 ; 93,1 ; 98,5$. 7. $16 ; 124,19$. 21. 24 ; 125,$8 ; 134,15 ; 137,17$.

Anders sind die Parallelen zum VC-Kontext in der Sekundärüberlieferung zu beurteilen, da sie direkt oder indirekt von der $\mathrm{VC}$ abhängen. Hier sind besonders einige der hagiographischen Schriften sehr hilfreich. An einigen Stellen sind diese Lesarten den von $b$ gebotenen überlegen: S. 26,$1 ; 31,19 ; 35,11 ; 94,24 ; 141,20$. Für die Werke der Kirchenhistoriker gilt das nur S. 88, 6. 11. Das nützlichste von ihnen ist für den Text der VC das Werk des Sokrates.

Wieder andere Maßstäbe sind an die Parallelen in anderen eusebianischen Werken anzulegen. Sie bieten nur eine bedingte Hilfe. Immerhin gibt es einige Stellen, an denen ihre Lesarten wegen Verderbtheit von $b$ in den Text aufgenommen wurden: S. 40, 5; 43, 24; 47,$10 ; 48,24 ; 109,14$.

Ein großer Teil der VC ist nur durch die Gruppe $b$ gestützt, ohne $\mathrm{da} B$ es eine Kontrollmöglichkeit durch andere Zeugen gibt. Obwohl

1 Der Vermutung S. 23,7 (Wil) kann ich nicht zustimmen. 
der Vergleich mit der Parallel- und Sekundärüberlieferung und die unverhältnismäßig vielen, im Laufe der Zeit gemachten Änderungsvorschläge verschiedener Gelehrter zeigen, daß diese Überlieferung nicht gut ist ${ }^{1}$, habe ich nach Möglichkeit den Text von $b$ belassen, solange er verständlich und nicht falsch ist ${ }^{2}$. Trotzdem war eine große Zahl von Änderungen nicht zu umgehen: S. 5,$4 ; 8,28 ; 9,24 ; 15,4.13$; 16,$15 ; 17,4$. 10 (bis). 11. $18 ; 18,6$. 7. 14. 18. $24 ; 21,20$. $26 ; 23,15$; 24,$21 ; 25,15 ; 27,4.17 ; 28,4 ; 31,3.12 ; 32,16.17 ; 34,6 ; 42,4$. 17. 29; 43,$19 ; 45,11 ; 46,5 ; 49,4$. 18. $21 ; 50,19 ; 52,23 ; 53,5 ; 55,20 ; 57,19$. $30 ; 62,24 ; 64,6 ; 68,8 ; 74,6$. $14 ; 80,17 ; 81.5$. 14. 20. 29. 31; 82,1. 2 . $3 ; 83,20 ; 85,14 ; 87,12.15 .16 ; 89,8.19 ; 96,27 ; 100,3$. 7. 29. 30. 32; $105,2.11$ (bis); 107, 20;110,23; 111, 3. 8; 113,4. 11. 14; 114, 5. 11. 27 ; 115,3.9. 17. $24 ; 119,2$. 11. $30 ; 122,26 ; 126,3.13 .19 ; 127,4.17$; 129,$15 ; 133,21 ; 136,2 ; 138,12.21 ; 139,20-23 ; 140,3.21 ; 141,3$; $143,7.12 ; 145,11$. 15. 20. $21 ; 146,1 ; 147,23 ; 150,18 ; 151,2$.

\section{Testimonia veterum}

Überlieferung und Testimonien sind - wenn man die späteren $\mathrm{Ab}$ schriften abrechnet - für solch ein im Hinblick sowohl auf den Verfasser als auch auf Gegenstand und Inhalt interessantes Werk wie die $\mathrm{VC}$ nicht sehr zahlreich. Bei den Testimonien basieren nur die Kirchenhistoriker des 4. und 5. Jahrhunderts, Gelasius von Caesarea, Socrates, Sozomenus und Theodoret, weiter der gelehrte Photios und vielleicht das Carmen Ebedjesu auf eigener Kenntnis der VC. Nur wenige der hagiographischen Viten haben Parallelen zur VC. Von diesen wenigen Viten wiederum haben nur zwei, nämlich $\mathrm{BHG} \mathrm{Nr}$. 363 und $369 \mathrm{~g}$, die VC direkt benutzt, während die anderen ihre Kenntnis aus den Kirchengeschichten des Gelasius von Caesarea und des Theodoret entnommen haben. Beide Viten sind in Konstantinopel entstanden; die eine auf Grund wissenschaftlicher Intentionen (BHG 363), die andere als persönliches Anliegen eines Schreibers oder eines Klosters (BHG 369g). Nur die erste dieser beiden Viten fand weitere Verbreitung. Die Handschriftengrundlage ist schmal; denn nur fünf Handschriften kommen für die Textkonstituierung in Frage, wovon nur eine - sie ist nicht die durchweg beste - aus dem 10. Jahrhundert, die anderen aus dem 12. Jahrhundert und späterer Zeit stammen. BHG $369 \mathrm{~g}$ ist nur in einer Handschrift überliefert.

Für diese schlechte Überlieferung und Bezeugung gibt es mehrere Gründe. Dem wichtigsten begegnen wir im Ansatz schon bei Sokrates (siehe unten S. XXIX), sehr deutlich dann in der Beurteilung derVCdurch

1 Einige der Fehler von $b$ können durchaus auf den Archetyp zurückgehen.

2 Siehe auch unten S. LXVI. 